\title{
特別講演
}

\section{NEW METHODS OF RESPIRATORY SUPPORT IN THE TREATMENT OF ACUTE LUNG DISEASE.}

\author{
Malcolm K. Sykes, M. B., B. Chir., F. F. A. R. A. C. S (Hon)*
}

There are now many techniques which can be used to provide a respiratory support in patients with acute lung disease. Most of the techniques in clinical use involve the application of positive pressure to the airway with a view to maintaining tidal volume and functional residual capacity.

In patients who have normal respiratory control mechanisms, the application of continuous positive pressure breathing can be used to increase lung volume. This achieves an increase in end-expiratory lung volume with a minimal decrease in cardiac output. However, with most commercially available breathing systems there is a marked reduction in the applied pressure during inspiration which greatly increases the work of breathing. To obviate this, it is necessary to minimize inspiratory resistance and to use very large reservoir bags or weighted bellows to supply the inspiratory gas flow.

The application of positive end-expiratory pressure during mechanical ventilation is the technique most commonly used to increase lung volume. However, this may produce severe falls in cardiac output with associated renal and hepatic failure, and may also result in barotrauma. Although blood-gases are usually improved, oxygen transport is frequently reduced unless great care is taken to support the circulation by appropriate increases in blood volume or inotropic support. All the evidence suggests that the application of positive end-expiratory pressure tends to increase rather than decrease lung water in patients with pulmonary edema, and there is also accumulating evidence that the use of PEEP is associated with the permanent dilatation of terminal airways.

Inversed ratio ventilation, in which inspiratory time exceeds expiratory time is now, frequently used as an alternative means of increasing lung volume. An increase in the duration of inspiration reduces physiological dead space and so improves $\mathrm{CO}_{2}$ elimination for a given tidal volume. However, the increase in lung volume associated with the use of this technique is critically dependent on lung compliance and airway resistance which govern the time 
constant for emptying of the lung. Small changes in airway resistance produce large changes in end-expiratory lung volume and it is, therefore, helpful to monitor changes of lung volume (e. g. by inductance plethysmography) when adjusting the ventilator. Although peak airway pressures may be reduced, the mean airway pressures required to produce a given increase in end-expiratory lung volume are similar to those measured during positive end-expiratory pressure ventilation so that there is usually an associated fall in cardiac output which can result in an over-all reduction in oxygen transport despite an improvement in arterial $\mathrm{PO}_{2}$.

Two alternative techniques have now been introduced to minimize the risk of barotrauma from high-airway pressures. The first of these is high-frequency ventilation. There are basically two techniques, one involving the use of a jet ventilator and the second high-frequency oscillation. Both these techniques can produce adequate $\mathrm{CO}_{2}$ removal with small tidal volumes and, hence, with reduced peak airway pressures. The reduction in peak pressure may reduce the risk of barotrauma and may also reduce intraregional stresses within the lung caused by regional differences in lung compliance. This may be of importance in preventing further lung damage. The reduced peak pressures also reduce the leak from a broncho-pleural fistula. However, although peak pressures are reduced, it is now apparent that the improvment in oxygenation often reported is due to gas trapping within the lung and an associated increase in lung volume - the so-called "alveolar PEEP". This increase in lung volume is again very dependent on small changes in airway resistance and continuous monitoring of end-expiratory lung volume is highly desirable. There is no evidence that for a given mean airway pressure the cardiac output is reduced any less than with any other technique of ventilation.

With high-frequency jet ventilation $\mathrm{CO}_{2}$ clearance for any given minute volume is greatest at low frequencies $(1-2 \mathrm{~Hz}$ ). At these frequencies gas exchange can be explained on the basis of conventional physiological principles. However, at higher frequencies $(3-5 \mathrm{~Hz})$ tidal volume is usually less than the anatomical dead space and $\mathrm{CO}_{2}$ elimination becomes very dependent on the distance of the jet from the alveoli.

High-frequency oscillation $(10-30 \mathrm{~Hz})$ is more difficult to apply because a bias flow of gas must be provided to clear $\mathrm{CO}_{2}$ and this results in a variable loss of gas from the oscillator. However, application of this technique in rabbits submitted to lung lavage improved survival, maintained arterial $\mathrm{PO}_{2}$ at normal levels, and reduced the formation of hyaline membranes, and it may well prove to be a useful technique in the treatment of neonates with the respiratory distress syndrome. Properly designed controlled trials are required to define the sphere of usefulness of these two techniques of high-frequency ventilation.

A randomized trial in the United States showed that there was no improvement in mortality when extracorporeal membrane oxygenation was used for the treatment of the adult re- 
spiratory distress syndrome. However, the modified technique of extracorporeal $\mathrm{CO}_{2}$ removal may prove to be of use. This technique differs from extracorporeal membrane oxygenation in that the membrane lung is used only to remove carbon dioxide by veno-venous perfusion, whilst oxygenation is achieved by apnoeic mass-transfer down the trachea, lung volume being maintained by allowing the oxygen to overflow through a positive end-expiratory pressure valve. This technique has several advantages.

(1) By separating the two processes of oxygenation and $\mathrm{CO}_{2}$ removal, it is possible to reduce the membrane blood flow to one $l / \mathrm{min}$, thus reducing blood trauma and minimizing difficulties in vascular access.

(2) The alveolar $\mathrm{PO}_{2}$ is determined by the $\mathrm{PO}_{2}$ in the membrane lung so that oxygen toxicity can be minimized.

(3) The membrane lung can be optimized for $\mathrm{CO}_{2}$ removal and can, therefore, be more efficient than a conventional membrane lung.

We have now developed a membrane lung which is capable of removing the total $\mathrm{CO}_{2}$ production with a blood flow of one $l / \mathrm{min}$ and a surface area of 0.9 sq.m. and priming volume of $280 \mathrm{ml}$. This high efficiency is achieved by use of the vortex-mixing principle which greatly enhances diffusion of gases within the blood. The clinical application of this technique in Milan has given promising results and there is now a need for properly controlled trials. 